\title{
Increased miR-146a in gastric cancer directly targets SMAD4 and is involved in modulating cell proliferation and apoptosis
}

\author{
BIN XIAO $^{1^{*}}$, EN-DONG ZHU ${ }^{1 *}$, NA LI $^{1}$, DONG-SHUI LU ${ }^{1}$, WEI LI ${ }^{2}$, BO-SHENG LI ${ }^{1}$, \\ YONG-LIANG ZHAO ${ }^{3}$, XU-HU MAO ${ }^{1}$, GANG GUO ${ }^{1}$, PEI-WU YU ${ }^{3}$ and QUAN-MING ZOU ${ }^{1}$ \\ ${ }^{1}$ Department of Clinical Microbiology and Immunology, College of Medical Laboratory Science, ${ }^{2}$ Department of Pharmacy, \\ Southwest Hospital, ${ }^{3}$ Department of General Surgery and Center of Minimally Invasive Gastrointestinal Surgery, \\ Southwest Hospital, Third Military Medical University, Chongqing 400038, P.R. China
}

Received August 11, 2011; Accepted September 19, 2011

DOI: $10.3892 /$ or.2011.1514

\begin{abstract}
MicroRNAs (miRNAs) have emerged as important gene regulators and are recognized as oncogenes or tumor suppressor genes in carcinogenesis. Gastric cancer is one of the most common malignant diseases worldwide. Our previous studies have revealed that miR-146a is upregulated in gastric epithelial cells infected with Helicobacter pylori (H.pylori) and in mucosal tissues from $H$. pylori-positive patients. However, the role of miR-146a in gastric cancer is largely unknown. In the current study, we showed that miR-146a was upregulated in 20 gastric cancer tissues compared with matched non-tumor adjacent tissues by quantitative RT-PCR. Furthermore, ectopic expression of miR-146a could improve cell proliferation in vitro by using Cell Counting kit 8 (CCK-8). We also found that miR-146a inhibited apoptosis of gastric cancer cells by flow cytometry (FCM) and Caspase-Glo ${ }^{\circledR}$ 3/7 assay. Using target prediction algorithms, luciferase reporter assay and Western blot assay, SMAD family member 4 (SMAD4) was identified as a target gene of miR-146a in gastric cancer. Moreover, an inverse correlation was observed between the expression of SMAD4 mRNA and miR-146a in gastric cancer tissues ( $\mathrm{R}=-0.731, \mathrm{P}=0.039$, Pearson's correlation). Taken together, our results provide important evidence that miR-146a can directly target SMAD4, and suggest that miR-146a may play a role in the
\end{abstract}

Correspondence to: Dr Quan-Ming Zou, Department of Clinical Microbiology and Immunology, College of Medical Laboratory Science, Third Military Medical University, Chongqing 400038, P.R. China

E-mail: qmzoutmmu@yeah.net

Dr Pei-Wu Yu, Department of General Surgery and Center of Minimally Invasive Gastrointestinal Surgery, Southwest Hospital, Third Military Medical University, Chongqing 400038, P.R. China E-mail: yu-peiwu@swhospital.com

*Contributed equally

Key words: microRNA, gastric cancer, miR-146a, SMAD4, proliferation, apoptosis development of gastric cancer by modulating cell proliferation and apoptosis. miR-146a could serve as a potential biomarker and therapeutic target against gastric cancer.

\section{Introduction}

Gastric cancer is one of the most common malignant diseases worldwide. A total of about 989,600 new gastric cancer cases and 738,000 deaths are estimated to have occurred each year, accounting for $8 \%$ of the total cases and $10 \%$ of total deaths (1). The development of gastric cancer is thought to be multifactorial, and Helicobacter pylori (H. pylori) infection seems to be the most important trigger (2). However, the molecular pathogenesis of gastric cancer still remains to be explored.

MicroRNAs (miRNAs) are small regulatory RNA molecules that post-transcriptionally modulate gene expression and play important roles in a wide variety of physiological and pathological processes, such as development, cell differentiation, apoptosis, and signal transduction (3). To date, 1048 human miRNAs have been identified (miRBase Release 16, September 2010). Growing evidence has shown that miRNAs are frequently dysregulated in human cancers and that miRNAs can function as oncogenes or tumor suppressor genes (4-6). Recently, a substantial number of deregulated miRNAs including miR-106b-25 cluster (7), miR-21 (8), miR-218 (9), miR-9 (10), and miR-222 (11) have been found in gastric cancer. These miRNAs may play a crucial role in the pathogenesis of gastric cancer through regulating cell proliferation, apoptosis, migration, or invasion.

Recent reports of our research and other studies have highlighted the regulatory role of miRNAs in $H$. pylori infection and associated diseases. In our previous study, we reported the distinct expression profile of miRNAs in human gastric epithelial cells infected with $H$. pylori, and miR-146a was one of the most up-regulated miRNAs (12). Further studies showed that miR-146a may function as a novel negative regulator to fine-tune H. pylori-induced inflammation (13). Recent evidence has indicated that miR-146a plays a key role in the negative regulation of the innate immune response, virus infection and autoimmune disorders (14). In particular, the deregulated expression of miR-146 has also been implicated in various cancers (15). However, many controversies still exist regarding the effects of 
miR-146a on cancer development and progression. To date, the role of miR-146a in gastric cancer is largely unknown.

In the present study, we found that miR-146a was significantly overexpressed in gastric cancer tissues. Ectopic expression of miR-146a could increase cell proliferation and inhibit apoptosis of gastric cancer cells in vitro. We also confirmed SMAD4 was a target gene of miR-146a in gastric cancer. Moreover, an inverse correlation was observed between the expression of SMAD4 and miR-146a in gastric cancer tissues. These results suggest that miR-146a may play a role in the development of gastric cancer by modulating cell proliferation and apoptosis.

\section{Materials and methods}

Cell lines and H. pylori culture. Human gastric cancer cell line BGC-823, SGC-7901, AGS, MKN-45, and human embryonic kidney HEK-293 cells were routinely cultured in RPMI-1640 medium (Invitrogen) supplemented with $10 \%$ fetal bovine serum (FBS), $100 \mathrm{U} / \mathrm{ml}$ penicillin. HGC-27 cells were cultured in DMEM medium (Invitrogen) containing 10\% FBS and $100 \mathrm{U} / \mathrm{ml}$ penicillin. All cells were maintained in a humidified incubator containing $5 \% \mathrm{CO}_{2}$ at $37^{\circ} \mathrm{C}$. H. pylori strain 26695 was obtained from ATCC, and was grown as previously described (12).

Tissue samples. Gastric cancer tissues and adjacent non-tumor tissues were obtained from patients with primary gastric cancer undergoing radical gastrectomy at Southwest Hospital, Third Military Medical University, China, and the clinicopathologic characteristics of gastric cancer patients are summarised in Table I. After surgical removal, the tissues were frozen immediately in liquid nitrogen and stored at $-80^{\circ} \mathrm{C}$. The study was approved by the ethics review board at Third Military Medical University, and informed consent was obtained from all patients before participation.

Quantitative RT-PCR. Quantitative RT-PCR (qRT-PCR) analysis for miR-146a was performed by using TaqMan miRNA assays (Ambion). Reverse transcription reactions were performed using the following parameters: $16^{\circ} \mathrm{C}, 30 \mathrm{~min}, 42^{\circ} \mathrm{C}$, $30 \mathrm{~min}$ and $84^{\circ} \mathrm{C}, 5 \mathrm{~min}$. PCR reactions were performed using the following parameters: $95^{\circ} \mathrm{C}$ for $2 \mathrm{~min}$ followed by 40 cycles of $95^{\circ} \mathrm{C}, 15 \mathrm{sec}$ and $60^{\circ} \mathrm{C}, 30 \mathrm{sec}$. A U6 small nuclear RNA was used as endogenous control for data normalization. Relative expression was calculated using the comparative threshold cycle (Ct) method.

qRT-PCR analysis for SMAD4 was performed by using PrimeScript RT-PCR kits (Takara). The mRNA level of $\beta$-actin was used as an internal control. The sequences of primers used are shown in Table II. The conditions for PCR were as follows: $95^{\circ} \mathrm{C}$ for $30 \mathrm{sec}(1 \mathrm{cycle}), 40$ cycles of $95^{\circ} \mathrm{C}$ for $5 \mathrm{sec}, 60^{\circ} \mathrm{C}$ for $5 \mathrm{sec}$, and $72^{\circ} \mathrm{C}$ for $30 \mathrm{sec}$.

Transient transfection of miR-146a mimics or inhibitors. miR-146a mimics, inhibitors and scrambled miR-control were obtained from GenePharma (Shanghai GenePharma Co. Ltd., China). Transfections were performed using Lipofectamine 2000 (Invitrogen). Cells were respectively transfected with miR-146a mimics, inhibitors, or miR-control at a final $50 \mathrm{nM}$ concentration.
Table I. Summary of clinicopathological parameters of patients with gastric cancer.

Clinicopathological parameters

No. of cases $(\%)$

\section{Gender \\ Male}

Female

Age

$\leq 60$

$>60$

Diameter $(\mathrm{cm})$

$\leq 5$

$>5$

Local invasion

$\mathrm{T} 1, \mathrm{~T} 2$

$\mathrm{T} 3, \mathrm{~T} 4$

Lymph node metastasis

Negative

Positive

TNM stage

I, II

III, IV

Construction of luciferase report vector. The construction of luciferase reporter vectors for miR-146a target, SMAD4, was performed according to the instructions and as previously described $(12,16)$. Complementary 56-mer DNA oligonucleotides containing the putative target site within the 3'UTR of SMAD4 mRNA were synthesized with flanking SpeI and HindIII restriction enzyme digestion sites. Another construct containing mutant seed region was also generated as a control (AGTTCTCA to TACCAGTC). The sequences are shown in Table II.

Luciferase assay. HEK-293 cells were transfected with $0.8 \mu \mathrm{g}$ of firefly luciferase reporter vector or mutant vector, $100 \mathrm{nM}$ miR-146a mimics, inhibitors or miR-control, and $0.04 \mu \mathrm{g}$ of Renilla luciferase control vector (pRL-TK-Promega) using Lipofectamine 2000 (Invitrogen). Luciferase assays were performed using the dual luciferase reporter assay system (Promega). Firefly luciferase activity was normalized to Renilla luciferase activity.

Cell proliferation assay. Briefly, cells were seeded at a density of $10^{4}$ per well in 96-well plates and transfected with miR-146a mimics or miR-control. Cell proliferation was assessed $48 \mathrm{~h}$ later using Cell Counting kit 8 (CCK8, Beyotime) according to manufacturer's protocol.

Western blot analysis. SMAD4 protein was analyzed by Western blot analysis using SMAD4 monoclonal antibody (Santa Cruz) with dillution 1:200 as previously described (13). 
Table II. Sequences of oligonucleotides used.

\begin{tabular}{ll}
\hline Name & \multicolumn{1}{c}{ Sequences } \\
\hline miR-146a mimics & 5'-UGAGAACUGAAUUCCAUGGGUU-3' \\
& 5'-CCCAUGGAAUUCAGUUCUCAUU-3' \\
miR-146a inhibitors & 5'-AACCCAUGGAAUUCAGUUCUCA-3' \\
SMAD4-sense & 5'-CTAGTGGGCCCTGATTTTAAAGGCAGAGAAGTTCTCAAAGTTAATTCACCTATGTA-3' \\
SMAD4-antisense & 5'-AGCTTACATAGGTGAATTAACTTTGAGAACTTCTCTGCCTTTAAAATCAGGGCCCA-3' \\
SMAD4-mu-sense & 5'-CTAGTGGGCCCTGATTTTAAAGGCAGAGATACCAGTCAAGTTAATTCACCTATGTA-3' \\
SMAD4-mu antisense & 5'-AGCTTACATAGGTGAATTAACTTGACTGGTATCTCTGCCTTTAAAATCAGGGCCCA-3' \\
SMAD4-F & 5'-AGGATCAGTAGGTGGAATAGC-3' \\
SMAD4-R & 5'-TGCATAAGCGACGAAGG-3' \\
$\beta$-actin-F & 5'-TTCCTTCCTGGGCATGGAGTCC-3' \\
$\beta$-actin-R & 5'-TGGCGTACAGGTCTTTGCGG-3' \\
\hline
\end{tabular}

Apoptosis analysis. SGC-7901 cells were transfected with miR-146a or miR-control, and the medium was replaced with serum-free RPMI-1640 for $24 \mathrm{~h}$. Then cells were continued in culture in the presence or absence of cis-diaminodichloro platin (CDDP) for $24 \mathrm{~h}$ and cells were applied to apoptosis analysis. For apoptosis analysis, an Annexin V-FITC Apoptosis Detection kit I (BD Pharmingen) was used according to the manufacturer's instructions. The apoptosis was analysed by flow cytometry (BD FACSCanto $\left.^{\mathrm{TM}} \mathrm{II}\right)$.

Caspase-Glo ${ }^{\circledR}$ 3/7 assay. The activity of caspase-3/7 was detected using the Caspase-Glo 3/7 Assay (Promega) according to the manufacturer's instructions. Caspase-Glo 3/7 reagent $(100 \mu \mathrm{l})$ was added to a white-walled 96-well plate then gently mixed, and incubated at room temperature for $30 \mathrm{~min}$. The luminescence was detected using the GloMax ${ }^{\circledR}-96$ Microplate Luminometer (Promega).

Statistical analysis. The results are expressed as mean \pm SD from at least 3 separate experiments performed in triplicate. The differences between groups were determined using two-tailed Student's t-test. The relationship between the miR-146a level and SMAD4 expression was analysed using Pearson's correlation. All statistical analyses were performed using SPSS 17.0 software. $\mathrm{P}<0.05$ were considered significant.

\section{Results}

miR-146a is frequently up-regulated in gastric cancer tissues. To explore the role of miR-146a in gastric cancer, we analyzed the expression of miR-146a in gastric cancer tissues by using qRT-PCR. Among a total of 20 paired gastric cancer tissues and adjacent non-tumor gastric tissues, 75\% (15/20) of cases showed up-regulation of miR-146a. As shown in Fig. 1B, we also found that the mean miR-146a level was significantly up-regulated in tumor tissues compared with matched non-tumor tissues $(6.46$ versus $2.79, \mathrm{P}<0.01$ ). We also examined the miR-146a expression in the gastric cancer cell lines BGC-823, MKN-45, HGC-27, AGS, and SGC-7901 compared with the non-malignant gastric epithelial cell line GES-1 and three normal gastric mucosa tissues. However, to our surprise, the expression of miR-146a was substantially down-regulated (Fig. 1C). One possible explanation might be the loss of miR-146a in the long-term adaption process of gastric cancer cell lines, and the contradiction in miR-146a expression was also found between melanoma patients and cell lines (17).

H. pylori infection increases the expression of $\mathrm{miR}-146 \mathrm{a}$ in gastric cancer cells. Because $H$. pylori infection seems to be an important factor for gastric cancer, we previously reported that H. pylori stimuli was able to induce the up-regulation of miR146a in many types of gastric epithelial cell lines including AGS, MKN-45 and HGC-27. Here we extend this observation to other gastric cancer cell lines. As shown in Fig. 2, H. pylori resulted in the increase of miR-146a in BGC-823 and SGC-7901, 2.77- and 4.47-fold change respectively $(\mathrm{P}<0.01)$. These data suggest that overexpression of miR-146a in gastric cancer tissues might be related to $H$.pylori infection.

Overexpression of miR-146a increases cell proliferation and inhibits apoptosis of gastric cancer cells in vitro. To deter-mine the potential role of miR-146a in gastric cancer, we examined the effect of miR-146a on tumor cell proliferation. AGS, HGC-27 and SGC-7901 cells were transfected with miR-146a mimics or scrambled miR-control for $48 \mathrm{~h}$, and the validity of miR-146a ectopic expression was confirmed by qRT-PCR. As shown in Fig. 3A, miR-146a level was obviously increased in cells transfected with miR-146a mimics. The effect of miR-146a on cell proliferation was evaluated by using CCK8 assay. We observed a significant increase in proliferation after transfection of miR-146a (Fig. 3B). Above data suggest that miR-146a can enhance cell proliferation in vitro.

Then we further investigated the effect of miR-146a on the apoptosis of gastric cancer cell. It is well accepted that nutrition starvation can induce apoptosis, therefore the effect of miR-146a on apoptosis of serum deprived SGC-7901 cells was evaluated by FCM analysis and caspase-3/7 activity assays. SGC-7901 cells were transfected with miR-146a or miR-control, cells were deprived of serum for $48 \mathrm{~h}$ before FCM examination. Compared with miR-control-transfected cells, the proportion of apoptotic 
A

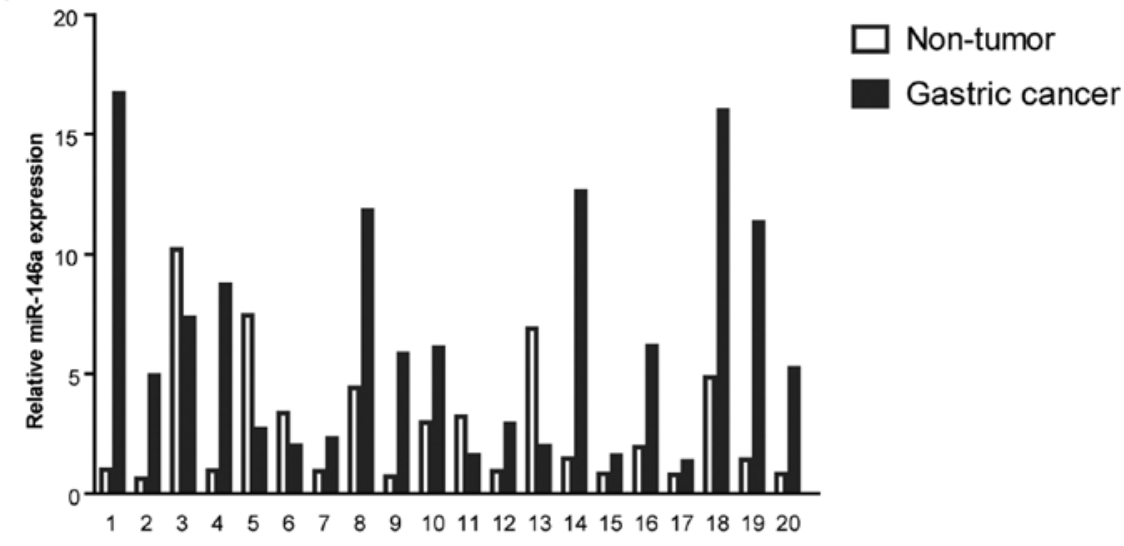

B

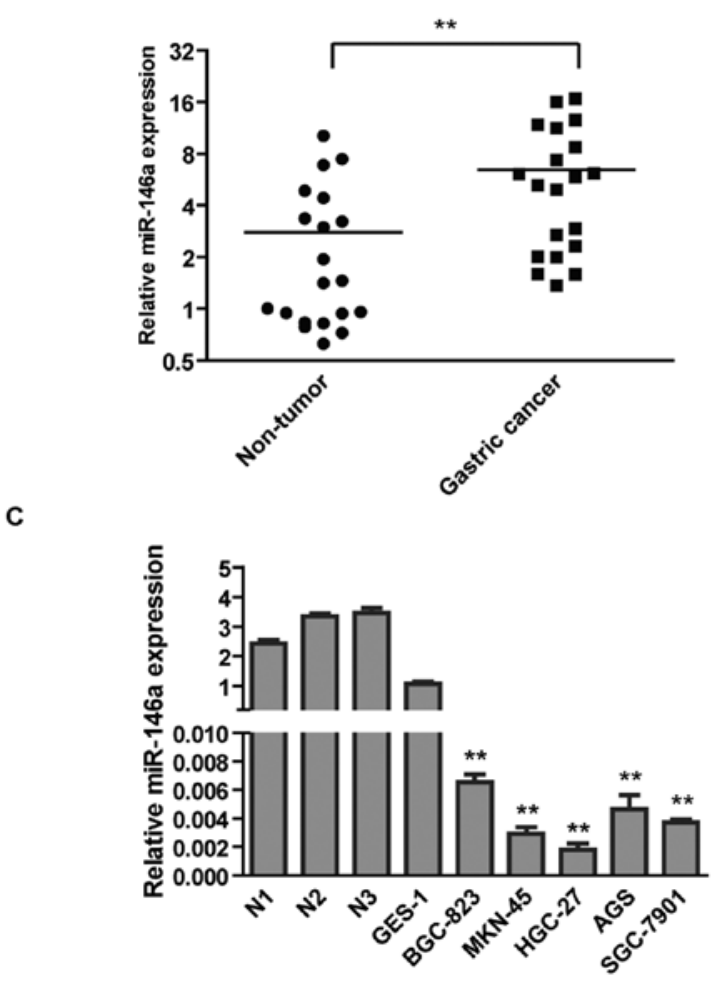

Figure 1. The expression of miR-146a in gastric cancer tissues and cell lines. (A) Relative expression of miR-146a in 20 gastric cancer tissues compared with matched adjacent non-tumor gastric tissues. (B) Comparison of the average expression level of miR-146a between gastric cancer tissues and non-tumor gastric tissues. ${ }^{* *} \mathrm{P}<0.01$. (C) Relative expression of miR-146a in gastric cancer cell lines compared with the non-malignant gastric epithelial cell line GES-1 and three normal gastric mucosa tissues. Data are mean \pm SD of triplicate samples obtained from a single representative experiment of three separate experiments. ${ }^{* *} \mathrm{P}<0.01$, compared with GES-1.

cells after transfection with miR-146a mimics was significantly decreased (22.63\% versus $10.37 \%$ ). To further clarify the inhibition effect of miR-146a on apoptosis, we used CDDP as positive control, which is a common anti-tumor drug through inducing tumor cell apoptosis. SGC-7901 cells were transfected with miR146a or miR-control, followed by CDDP treatment $(5 \mu \mathrm{g} / \mathrm{ml})$. As shown in Fig. 3C, CDDP treatment increased the numbers of early and late apoptosis cells, whereas overexpression of miR146a significantly inhibited CDDP-induced apoptosis compared to the cells transfected with miR-control $(44.36 \%$ versus $15.09 \%$ ). Furthermore, obvious decrease in caspase-3/7 activity was found in miR-146a-transfected cells compared with miRcontrol transfectants (Fig. 3D, $\mathrm{P}<0.01$ ). Taken together, above

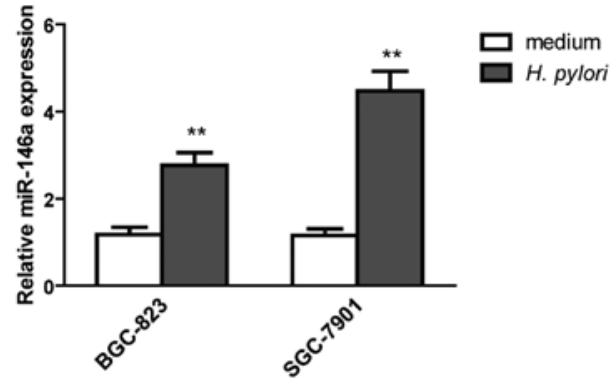

Figure 2. Up-regulation of miR-146a expression in gastric cancer cells infected with H.pylori. BGC-823 and SGC-7901 were infected with or without H.pylori stain 26695 at MOI 100 for $24 \mathrm{~h}$. Data are mean \pm SD of triplicate samples obtained from a single representative experiment of three separate experiments. ${ }^{* *} \mathrm{P}<0.01$. 
^

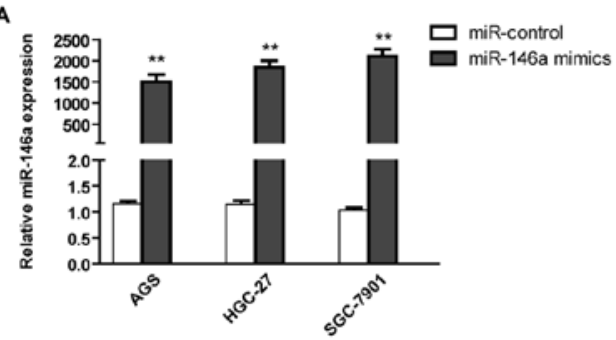

B

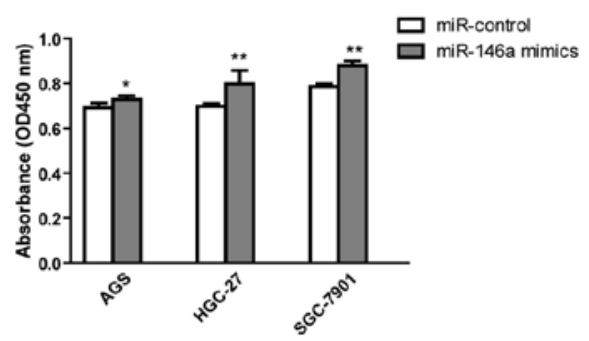

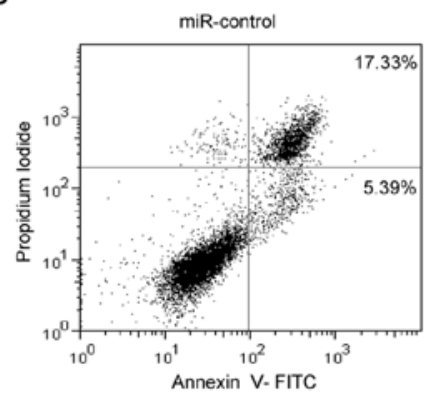

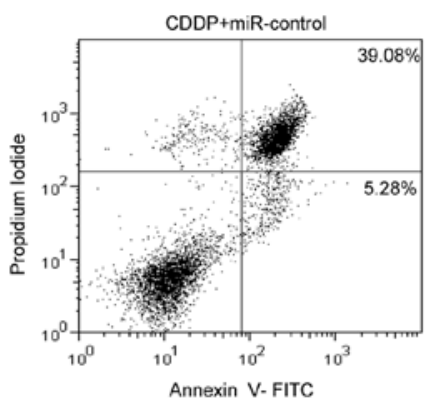

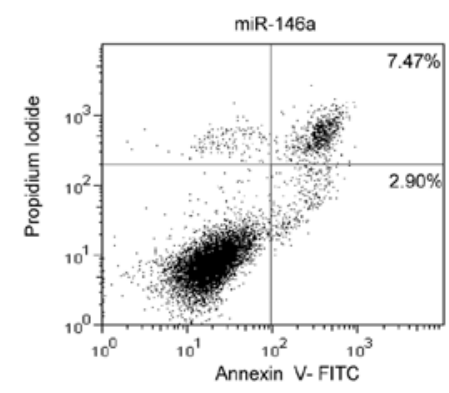

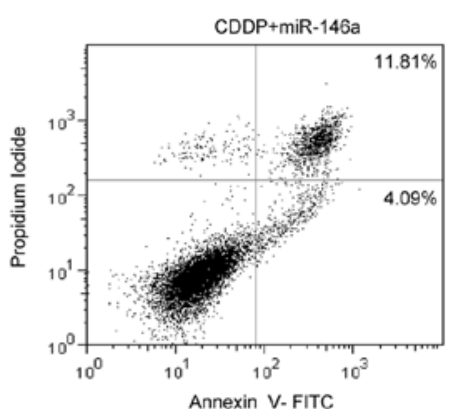

D

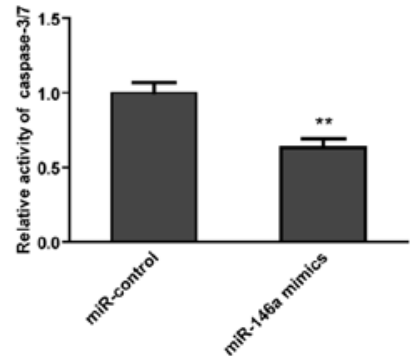

Figure 3. The effect of miR-146a on the proliferation and apoptosis of gastric cancer cells in vitro. (A) Relative expression of miR-146a in AGS, HGC-27 and SGC-7901 cells transfected with miR-146a mimics or miR-control. (B) Cell proliferation was measured by CCK-8 assay. AGS, HGC-27 and SGC-7901 cells were transfected with miR-146a mimics or miR-control $(50 \mathrm{nM})$ for $48 \mathrm{~h}$. Data are mean \pm SD of 6 duplications. ${ }^{* *} \mathrm{P}<0.01$. (C) SGC-7901 cells were transfected with miR-146a or miR-control, and the medium was replaced with serum-free RPMI-1640 for $24 \mathrm{~h}$. Then cells were continued in culture in the presence or absence of $\operatorname{CDDP}(5 \mu \mathrm{g} / \mathrm{ml})$ for $24 \mathrm{~h}$ and cells were applied to apoptosis analysis. (D) SGC-7901 cells were transfected with miR-146a mimics or miR-control (50 $\mathrm{nM}$ ) for $48 \mathrm{~h}$. The activity of caspase-3 and caspase-7 was detected using the Caspase-Glo 3/7 assay. Data are mean \pm SD of 6 duplications.

results indicate that miR-146a may function as an anti-apoptotic factor in human gastric cancer cells.

SMAD4 is a target of miR-146a. Because miR-146a has a potential oncogenic role in gastric cancer, it is important to identify the target gene of miR-146a. We searched for its potential target genes that exhibit tumor suppressor properties using different prediction algorithms such as TargetScan and PicTar. Among the potential targets of miR-146a, one interesting hit is SMAD4. As shown in Fig. 4A, SMAD4 mRNA 3'UTR has a putative miR-146a binding site, which was reversed complementary with the miR-146a 'seed region'. In addition, SMAD4 was confirmed as a target gene of miR-146a in the promyelocytic leukemia cell line NB4 (18). Therefore, in the current study, we further determined whether SMAD4 gene was an authentic target gene of miR-146a in gastric cancer. To directly address whether miR-146a binds to the 3'-UTR of target mRNA, we generated a luciferase reporter vector that contain the SMAD4 3'-UTR with the putative miR-146a binding sites. Correspondingly, we also generated a mutant reporter vector which contains the SMAD4 3'-UTR with a mutation at the putative miR-146a binding site. As shown in Fig. 4B, we observed a marked reduction in luciferase activity in cells transfected with miR-146a mimics compared with miR-control-transfected cells $(\mathrm{P}<0.01)$. In contrast, no change of luciferase was observed in cells transfected with the mutant 3 '-UTR constructs. We next determined 
A

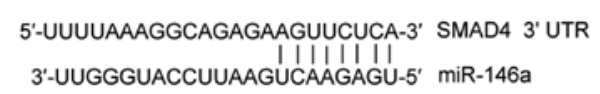

B

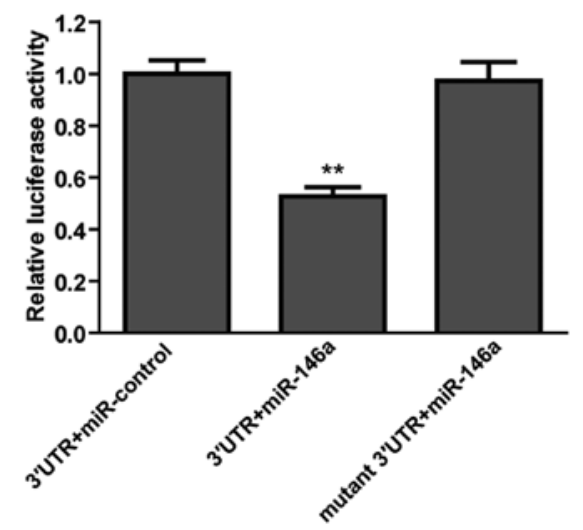

C

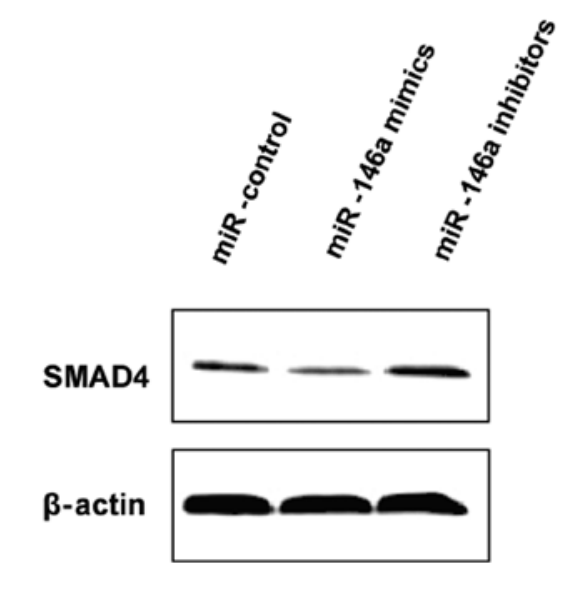

Figure 4. Identification of SMAD4 as a potential target of miR-146a in gastric cancer. (A) Sequence alignment of miR-146a and its target sites in 3'-UTRs of SMAD4. (B) HEK293 cells were transiently co-transfected with luciferase reporter vectors, and either miR-146a mimics or miR-control. Luciferase activities were normalized to the activity of Renilla luciferase. (C) SMAD4 protein in SGC-901 cells was detected by Western blot analysis after transfection with miR-146a mimics, inhibitors or miR-control (50 nM). $\beta$-actin was used as an internal loading control.

A
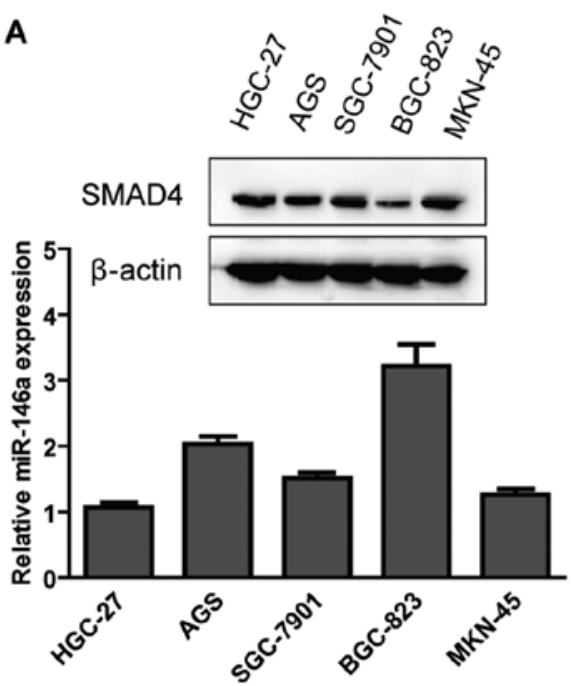

B

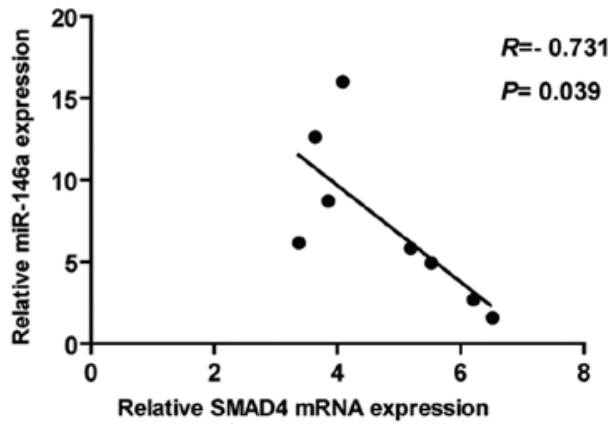

Figure 5. The miR-146a expression is inversely correlated with the expression of SMAD4 in gastric cancer. (A) The expression of miR-146a and SMAD4 protein in gastric cell lines. Top, Western blot analysis of SMAD4 protein levels; bottom, qRT-PCR analysis of miR-146a levels. (B) The correlation analysis between miR-146a and SMAD4 mRNA levels in gastric cancer tissues (Pearson's correlation analysis).

whether overexpression or knock-down of miR-146a can lead to down-regulation or up-regulation of SMAD4 protein expression. Western blot assays indicated that the SMAD4 protein level was obviously reduced in SGC-7901 cells overexpressing miR-146a compared with the control, whereas the SMAD4 level was up-regulated in cells transfected with miR-146a inhibitors. These results suggest that SMAD4 is a direct target of miR-146a in gastric cancer cells, and miR-146a may negatively regulate the expression of SMAD4.

The expression of miR-146a is inversely correlated with the expression of SMAD4 in gastric cancer. Given that miR-146a is up-regulated in gastric cancer and that SMAD4 is a target gene of miR-146a, and it has been reported that SMAD4 protein in gastric cancer tissues is dramatically lower than in the nontumor tissue (19). We hypothesized that reduced SMAD4 expression in gastric cancer could be a result of enhanced miR-146a expression. We initially examined SMAD4 protein and miR-146a levels in gastric cancer cell lines. As shown in Fig. 5A top, SMAD4 protein levels were higher in HGC-27, MKN-45, and SGC-7901 cells, while relative lower in BGC-823 and AGS cells. In contrast, miR-146a levels were relative lower in HGC-27, MKN-45, and SGC-7901 cells than in other cells (Fig. 5A bottom).

To further explore the correlation between miR-146a and SMAD4 expression in gastric cancer tissues, we examined miR-146a and SMAD4 mRNA in 10 sets of gastric cancer and matched normal tissues. We found that $80 \%(8 / 10)$ of gastric 
cancers displayed lower SMAD4 mRNA compared with normal tissues. As expected, miR-146a was commonly elevated in 8 of 10 tumors. Using Pearson's correlation analysis, we observed a significant inverse correlation between miR-146a and SMAD4 mRNA $(\mathrm{R}=-0.731, \mathrm{P}=0.039)$. Above results suggest that miR146a expression is inversely correlated with SMAD4 expression in gastric cancer.

\section{Discussion}

Accumulating evidence has indicated that dysregulation of miR-146a is associated with the development of cancers. However, the function of miR-146a in cancers is controversial. Elevated miR-146a expression has been found in cervical cancer (20), papillary thyroid carcinoma (21), breast cancer (22), and pancreas cancer (22). In contrast, miR-146a has also been found to be down-regulated in prostate cancer (23) and papillary thyroid carcinoma (24). In addition, miR-146a may function as a tumor-suppressor gene in prostate cancer and breast cancer through inhibiting invasion and metastasis $(25,26)$. These controversial results may reflect the diverse roles of miR-146a in different types of cancer.

To date, the expression level and the biological function of miR-146a in gastric cancer are still unclear. Here, we examined the expression of miR-146a in 20 paired gastric tumor tissues and non-tumor tissues, as well as five gastric cancer cell lines. Our results clearly demonstrated the up-regulation of miR-146a expression in gastric cancer tissues compared with non-tumor tissues. Interestingly, miR-146a was significantly down-regulated in gastric cancer lines compared with GES-1 and three normal gastric mucosa tissues. Consistent with our results, Philippidou et al (17) also found that miR-146a was up-regulated in human melanoma patients but none of the cell lines. The reason for the divergent expression patterns of miR-146a in patient tissues and cell lines was probably that long-term culture-induced adaption process results in the loss of miR-146a.

Regarding miR-146a level in gastric cancer, in line with our results, Tsukamoto et al (27) analyzed the expression profiles of miRNAs in gastric cancer by use of microarray, and found miR-146a was obviously up-regulated in cancer (8.86-fold change). In addition, Kim et al (11) also indicated enhanced miR-146a level in gastric cancer. However, recently, Hou et al (28) reported different expression of miR-146a in gastric cancer tissues. They found that 36 of 43 gastric cancer tissue samples (84\%) showed decreased expression of miR-146a. The discrepancies in miR-146a expression might be due to differences in sample origin, tumor clinicopathological characteristics or different technical platforms of the studies. We noted that all gastric cancer tissues in the study of Hou et al were formaldehyde-fixed, paraffin-embedded tissues. Thus far, several miRNAs with different expression patterns in the same cancer have been reported. For instance, miR-150 is up-regulated in various types of cancers, including gastric cancer and cervical cancer, while down-regulation of miR-150 expression has been found in undifferentiated gastric cancer (29). It is also possible that miR-146a has a dual role in tumorigenicity. These data add to the complexity of the miR-146a function in gastric cancer.

Given that miR-146a was up-regulated in gastric cancer tissues, we speculated that miR-146a might have an oncogenic effect in gastric cancer. As we expected, ectopic expression of miR-146a improved the cell proliferation of AGS, HGC-27 and SGC-7901. Similarly, introduction of miR-146a into cervical cancer cell lines increased cell proliferation (20). It is known that apoptosis is a major barrier that must be circumvented during cancer development. In this study, the effect of miR-146a on apoptosis of gastric cancer cells was evaluated by FCM and caspase-3/7 activity assays. Our results indicated that overexpression of miR-146a can inhibit apoptosis of gastric cancer cells. The above data suggest that miR-146a is involved in modulating cell proliferation and apoptosis in gastric cancer.

To explore the molecular mechanism underlying miR-146a function, it is important to identify its target gene. Recently, several novel targets of miR-146a have been confirmed including interleukin-1 receptor-associated kinase 1 (IRAK1), TNF receptor-associated factor 6 (TRAF6) (30), toll-like receptor 4 (TLR4) (31), signal transducer and activator transcription 1 (Stat1) (32), tata binding protein (33), and chemokine CCL8/ MCP-2 (34). We try to find a target gene which is involved in cancer development, and SMAD4 is an interesting candidate. Several pieces of evidence in our study indicate that SMAD4 is a direct target gene of miR-146a in gastric cancer. Firstly, overexpression of miR-146a significantly reduces the activity of a luciferase reporter containing the 3'UTR sequence of SMAD4. Secondly, overexpression of miR-146a down-regulates SMAD4 protein expression. Thirdly, miR-146a expression is inversely correlated with SMAD4 expression in gastric cancer cell lines and gastric tumors. In addition, SMAD4 has been confirmed as a target gene of miR-146a in promyelocytic leukemia cells (18). It is known that SMAD4 is the primary signaling pathway downstream of transforming growth factor- $\beta$ (TGF- $\beta$ ). Several lines of evidence have suggested that SMAD4 can function as a tumor suppressor gene in gastrointestinal carcinoma. Inactivation of SMAD4 occurs frequently in gastric cancer and is associated with gastric cancer progression $(19,35)$. Our data indicate that miR-146a may negatively regulate the expression of SMAD4 gene. Therefore, dysregulation of miR-146a may be another mechanism leading to down-regulation or loss of SMAD4 in gastric cancer.

$H$. pylori infection is the leading cause of gastric cancer, however the exact mechanisms of $H$. pylori carcinogenesis require further study. In the current study, we found that miR146a was up-regulated in gastric cancer cells infected with H. pylori and in gastric cancer tissues. In our previous study, elevated miR-146a levels were found in gastric mucosal tissues from $H$. pylori-positive patients. Furthermore, Zhang et al (8) have shown that miR-21 is overexpressed in H.pylori-infected gastric mucosa and gastric cancer tissues. These results suggest that miRNAs including miR-146a and miR-21 may act as a bridge between $H$. pylori and gastric cancer.

In conclusion, this study provides new insights into the role of miR-146a in gastric cancers. It shows that miR-146a is up-regulated in gastric cancer tissues and overexpression of miR-146a can improve cell proliferation and inhibit the apoptosis of gastric cancer cell in vitro. miR-146a can negatively regulate the expression of SMAD4 gene. These results suggest that miR-146a may function as a potential oncogene in gastric cancer, and indicate the potential application of miR-146a in cancer therapy. 


\section{Acknowledgements}

This study was supported by the Scientific Innovation Research Foundation of Third Military Medical University (no. 2009XQN20), Chinese National Natural Science Foundation project (no. 30770113), National Basic Research Program of China (973 Program) (no. 2009CB522606).

\section{References}

1. Jemal A, Bray F, Center MM, Ferlay J, Ward E and Forman D: Global cancer statistics. CA Cancer J Clin 61: 69-90, 2011.

2. Bornschein J, Rokkas T, Selgrad M and Malfertheiner P: Helicobacter pylori and clinical aspects of gastric cancer. Helicobacter 14 (Suppl. 1): S41-S45, 2009.

3. Bartel DP: MicroRNAs: genomics, biogenesis, mechanism, and function. Cell 116: 281-297, 2004.

4. Lu J, Getz G, Miska EA, et al: MicroRNA expression profiles classify human cancers. Nature 435: 834-838, 2005.

5. Esquela-Kerscher A and Slack FJ: Oncomirs - microRNAs with a role in cancer. Nat Rev Cancer 6: 259-269, 2006.

6. Croce CM: Causes and consequences of microRNA dysregulation in cancer. Nat Rev Genet 10: 704-714, 2009.

7. Petrocca F, Visone R, Onelli MR, et al:E2F1-regulated microRNAs impair TGFbeta-dependent cell-cycle arrest and apoptosis in gastric cancer. Cancer Cell 13: 272-286, 2008.

8. Zhang Z, Li Z, Gao C, et al: miR-21 plays a pivotal role in gastric cancer pathogenesis and progression. Lab Invest 88: 1358-1366, 2008.

9. Tie J, Pan Y, Zhao L, et al: MiR-218 inhibits invasion and metastasis of gastric cancer by targeting the Robol receptor. PLoS Genet 6: e1000879, 2010

10. Wan HY, Guo LM, Liu T, Liu M, Li X and Tang H: Regulation of the transcription factor NF-kappaB1 by microRNA-9 in human gastric adenocarcinoma. Mol Cancer 9: 16, 2010.

11. Kim YK, Yu J, Han TS, et al: Functional links between clustered microRNAs: suppression of cell-cycle inhibitors by microRNA clusters in gastric cancer. Nucleic Acids Res 37: 1672-1681, 2009.

12. Xiao B, Liu Z, Li BS, et al: Induction of microRNA-155 during Helicobacter pylori infection and its negative regulatory role in the inflammatory response. J Infect Dis 200: 916-925, 2009.

13. Liu Z, Xiao B, Tang B, et al: Up-regulated microRNA-146a negatively modulate Helicobacter pylori-induced inflammatory response in human gastric epithelial cells. Microbes Infect 12: 854-863, 2010.

14. Sonkoly E, Stahle M and Pivarcsi A: MicroRNAs and immunity: novel players in the regulation of normal immune function and inflammation. Semin Cancer Biol 18: 131-140, 2008.

15. Williams AE, Perry MM, Moschos SA, Larner-Svensson HM and Lindsay MA: Role of miRNA-146a in the regulation of the innate immune response and cancer. Biochem Soc Trans 36: 1211-1215, 2008 .

16. Chen XM, Splinter PL, O'Hara SP and LaRusso NF: A cellular micro-RNA, let-7i, regulates Toll-like receptor 4 expression and contributes to cholangiocyte immune responses against Cryptosporidium parvum infection. J Biol Chem 282: 28929-28938, 2007.

17. Philippidou D, Schmitt M, MoserD, et al: Signatures of microRNAs and selected microRNA target genes in human melanoma. Cancer Res 70: 4163-4173, 2010.
18. Zhong H, Wang HR, Yang S, et al: Targeting Smad4 links microRNA-146a to the TGF-beta pathway during retinoid acid induction in acute promyelocytic leukemia cell line. Int J Hematol 92: 129-135, 2010.

19. Wang LH, Kim SH, Lee JH, et al: Inactivation of SMAD4 tumor suppressor gene during gastric carcinoma progression. Clin Cancer Res 13: 102-110, 2007.

20. He H, Jazdzewski K, Li W, et al: The role of microRNA genes in papillary thyroid carcinoma. Proc Natl Acad Sci USA 102: 19075-19080, 2005.

21. Wang X, Tang S, Le SY, et al: Aberrant expression of oncogenic and tumor-suppressive microRNAs in cervical cancer is required for cancer cell growth. PLoS One 3: e2557, 2008.

22. Volinia S, Calin GA, Liu CG, et al: A microRNA expression signature of human solid tumors defines cancer gene targets. Proc Natl Acad Sci USA 103: 2257-2261, 2006.

23. Lin SL, Chiang A, Chang D and Ying SY: Loss of mir-146a function in hormone-refractory prostate cancer. RNA 14: 417-424, 2008.

24. Jazdzewski K, Murray EL, Franssila K, Jarzab B, Schoenberg DR and de la Chapelle A: Common SNP in pre-miR-146a decreases mature miR expression and predisposes to papillary thyroid carcinoma. Proc Natl Acad Sci USA 105: 7269-7274, 2008.

25. Hurst DR, Edmonds MD, Scott GK, Benz CC, Vaidya KS and Welch DR: Breast cancer metastasis suppressor 1 up-regulates miR-146, which suppresses breast cancer metastasis. Cancer Res 69: 1279-1283, 2009.

26. Bhaumik D, Scott GK, Schokrpur S, Patil CK, Campisi J and Benz CC: Expression of microRNA-146 suppresses NF-kappaB activity with reduction of metastatic potential in breast cancer cells. Oncogene 27: 5643-5647, 2008.

27. Tsukamoto Y, Nakada C, Noguchi T, et al: MicroRNA-375 is downregulated in gastric carcinomas and regulates cell survival by targeting PDK1 and 14-3-3zeta. Cancer Res 70: 2339-2349, 2010.

28. Hou Z, Xie L, Yu L, Qian X and Liu B: MicroRNA-146a is downregulated in gastric cancer and regulates cell proliferation and apoptosis. Med Oncol: Feb 24, 2011 [Epub ahead of print].

29. Katada T, Ishiguro H, Kuwabara Y, et al: microRNA expression profile in undifferentiated gastric cancer. Int J Oncol 34: 537-542, 2009.

30. Taganov KD, Boldin MP, Chang KJ and Baltimore D: NF-kappaBdependent induction of microRNA miR-146, an inhibitor targeted to signaling proteins of innate immune responses. Proc Natl Acad Sci USA 103: 12481-12486, 2006.

31. Yang K, He YS, Wang XQ, et al: MiR-146a inhibits oxidized low-density lipoprotein-induced lipid accumulation and inflammatory response via targeting toll-like receptor 4. FEBS Lett 585: 854-860, 2011.

32. Lu LF, Boldin MP, Chaudhry A, et al: Function of miR-146a in controlling Treg cell-mediated regulation of Th1 responses. Cell 142: 914-929, 2010.

33. Sinha M, Ghose J, Das E and Bhattarcharyya NP: Altered microRNAs in STHdh(Q111)/Hdh(Q111) cells: miR-146a targets TBP. Biochem Biophys Res Commun 396: 742-747, 2010.

34. Rom S, Rom I, Passiatore G, et al: CCL8/MCP-2 is a target for mir-146a in HIV-1-infected human microglial cells. FASEB J 24: 2292-2300, 2010.

35. Powell SM, Harper JC, Hamilton SR, Robinson CR and Cummings OW: Inactivation of Smad4 in gastric carcinomas. Cancer Res 57: 4221-4224, 1997. 